\title{
Nonlinear Classical Elasticity Model for Materials with Fluid and Matrix Phases
}

\author{
Rafael Muñoz $\mathbb{D}^{1,2}$ and Juan Melchor $\mathbb{D}^{2,3,4}$ \\ ${ }^{1}$ Department of Civil Engineering, University of Granada, Granada 18071, Spain \\ ${ }^{2}$ NDE Lab., Department of Structural Mechanics, University of Granada, Granada 18071, Spain \\ ${ }^{3}$ Biosanitary Research Institute, Granada 18071, Spain \\ ${ }^{4}$ MNat Scientific Unit of Excellence, University of Granada, Granada, Spain \\ Correspondence should be addressed to Rafael Muñoz; rmb@ugr.es
}

Received 14 September 2018; Accepted 1 November 2018; Published 27 November 2018

Academic Editor: John D. Clayton

Copyright (C) 2018 Rafael Muñoz and Juan Melchor. This is an open access article distributed under the Creative Commons Attribution License, which permits unrestricted use, distribution, and reproduction in any medium, provided the original work is properly cited.

\begin{abstract}
Materials with fluid and matrix phases present different acoustic responses in each phase. While longitudinal waves propagate in both phases, shear waves do it only through the solid matrix. Longitudinal waves are mainly described by volumetric propagation and shear waves by deviatoric processes. In the case of nonlinear propagation cross effects occur between both components. This paper presents a new classical nonlinear model proposing a constitutive equation that separates volumetric and deviatoric effects. Four nonlinear constants of third order are defined. The formulation is compared to constitutive equations with Landau constants for weakly elasticity and both types of nonlinear constants related. Some reinterpretation of the Landau's constants arises in terms of parallel or cross nonlinear effects between volumetric and deviatoric components.
\end{abstract}

\section{Introduction}

The study of the coefficients of acoustic nonlinearity has recently been recovered again [1-6]. The classical nonlinear behavior of a material can be characterized using different parameters: first-second nonlinear parameters, Landau and Murnaghan constants, and stiffness matrices of second and third order [7-9]. An example of this interest could be the advances in early damage detection [10-13] of materials with complex structures in Nondestricutive Evaluation field, allowing new paradigms that explore the connection between the micromechanical scales.

On the mathematical description of nonlinear effects, it is important to separate the geometrical nonlinearity from the material-dependent constitutive nonlinearity. The nonlinear analysis is based on Taylor series expansions of the strain energy, where the Landau [14] and Murnaghan [15] coefficients surge and only account for the materialdependent constitutive nonlinearity. The dynamic problem and its wave equation deduction bring several calculations that introduce geometrical nonlinearities. The first one arises when the stress tensor is calculated from the strain energy expansion, where several operations involving the deformation gradient tensor generate new nonlinear terms (see (13), terms that are independent of Landau constants). The second, the kinematic relations add the well-known cross derivative term of the displacements, which is independent on the material nonlinear behavior.

The understanding of the nonlinear constants meaning (Landau, Murnaghan, $\beta, \delta$, or stiffness coefficients) turns tough, and their interpretation does not appear as straight forward as it could seem. Probably, the most intuitive interpretation comes from the first nonlinear parameter $\beta$ and the Finite Amplitude technique [16], where the parameter is a measurement of the growth on the second harmonic amplitude with distance. However, this parameter must be further carefully interpreted, since different definitions of them can be found in literature while nomenclature is maintained the same $[10,17]$. As some examples in this vain, first and second nonlinear parameters $(\beta$ and $\delta$ ), defined directly as wave equation coefficients [18], usually accounts for both geometrical and constitutive nonlinearity. There are simplifications on some developments that define only constitutive versions 
of the coefficients $\beta$ and $\delta$, directly applying a Taylor series expansion on the stress as a function of strain that are used for unidimensional plane wave propagation cases and neglecting geometrical nonlinearity $[19,20]$. Anyway, all these theoretical proposals have definitely contributed to understand the main nonlinear experimental phenomena. But new approaches can better interpret different situations and open new visions.

This paper proposes deepening on the study of classical nonlinear acoustics, proposing a new mathematical formulation with the aim of presenting a different classical nonlinear equation. It splits the general first-order nonlinearity (usually measured by $\beta$ or by Landau's constants) into four specific nonlinear phenomena (measured with four new nonlinear constants), due to the interaction of the deviatoric and volumetric components of the deformation and stress tensors. This paradigm turns specially useful to explain shear wave nonlinear propagation in materials constituted by two phases, as solid fibers embedded in a quasi-fluid matrix. This is the case of soft tissues, where the propagation of shear waves in the liquid phase can be neglected against that occurring in the solid structure.

Nonlinear first- and second-order constants are usually referred to the nonlinear order of the terms in the wave equation, or in other cases at the constitutive equation, while other nonlinear constants are referred to the order of the Taylor series expansion of the Energy, like Landau constants as third order. As the new four nonlinear constants are compared to Landau constants in this paper, they will be referred as third-order nonlinear constants.

The concept would be scalable to the third and higher harmonics [21-23]. Non-classical nonlinear effects are not considered at the moment, in order to ease the analysis, and viscosity, just partially, but they could be considered in future works, since they could be noteworthy in some cases. The connection of these new four nonlinear constants with the Landau third-order elasticity constants is also explored.

\section{Methods}

The general method of this paper is the deduction of the nonlinear wave equation, where the new proposed constitutive equation is the starting point.

$$
\sigma_{i j}=\sigma_{i j}\left(v, D_{i j}\right)
$$

where $v$ and $D_{i j}$ are the volumetric and deviatoric parts of the deformation tensor $\varepsilon_{i j}$. This equation defines the new four $\beta$ nonlinear constants. The constitutive equation is transformed into $\sigma_{i j}=\sigma_{i j}\left(\varepsilon_{i j}\right)$ and compared with the similar expression given by Landau formulation for weakly elasticity to relate the four $\beta$ constants to Landau nonlinear constants.

Finally, the nonlinear equation of motion as a function of the new paremeters is deducted,

$$
\rho \ddot{u}_{i}=\sigma_{i j, j}
$$

2.1. Theoretical Preliminars. The dynamic elastic problem comprises the momentum balance equation, compatibility
TABLE 1: List of variables.

\begin{tabular}{lcc}
\hline Quantity & Symbol & Units \\
\hline Displacement & $u_{i}$ & $\mathrm{~m}$ \\
Stress & $\sigma_{i j}$ & $\mathrm{~Pa}$ \\
Strain & $\varepsilon_{i j}$ & - \\
Space & $x_{i}$ & $\mathrm{~m}$ \\
Time & $t$ & $\mathrm{~s}$ \\
Lamé constants & $\lambda, \mu$ & $\mathrm{Pa}$ \\
Density & $\rho$ & $\mathrm{kg} / \mathrm{m}^{3}$ \\
Kinematic viscosity & $\gamma=\frac{\eta}{\rho}$ & $\mathrm{s}^{-1}$ \\
\hline
\end{tabular}

equations, and the kinematic relationships. For wave propagation (body forces neglected), the first and third equations would be

$$
\begin{aligned}
\rho \ddot{u}_{i}+\gamma \rho \dot{u}_{i} & =\sigma_{i j, j} \\
\varepsilon_{i j} & =\frac{1}{2}\left(u_{i, j}+u_{j, i}+u_{k, i} u_{k, j}\right)
\end{aligned}
$$

where Table 1 shows the symbols of the magnitudes.

The compatibility equations can be considered separating them into linear, viscous, and nonlinear stress components, $\sigma_{i j}^{L}, \sigma_{i j}^{V}$, and $\sigma_{i j}^{N L}$.

$$
\sigma_{i j}=\sigma_{i j}^{L}+\sigma_{i j}^{V}+\sigma_{i j}^{N L}
$$

The nonlinear term can be deducted, following a similar concept of series expansion put forth by Landau [24, 25], where the volumetric and deviatoric decomposition of the stress tensor was considered, and only the volumetric part was detailed in terms of the nonlinear parameter $\beta$, in the following expression:

$$
-p=-3 K v+9 K \beta v^{2}-3 \eta^{v} \dot{v}
$$

where $p$ and $v$ are the volumetric components of the stress and deformation tensors, respectively, so that

$$
\begin{aligned}
& \sigma_{i j}=\underbrace{-p \delta_{i j}}_{\text {volumetric }}+\underbrace{\tau_{i j}}_{\text {deviatoric }}, \quad p=-\frac{1}{3} \sigma_{k k} \\
& \varepsilon_{i j}=-v \delta_{i j}+D_{i j}, \quad v=-\frac{1}{3} \varepsilon_{k k}
\end{aligned}
$$

with $\tau_{i j}$ and $D_{i j}$ being the deviatoric parts of the stress and strain tensors. 
2.2. Proposal of a New Constitutive Equation. However, a more generalized constitutive relationship can be hypothesized using a combination of four of nonlinear parameters of third-order $\beta$ that may explain a different scenario of calculations. These combinations could be expanded as exploring the whole set of combinations by quadratic terms as follows:

$$
\begin{gathered}
\sigma_{i j}=\underbrace{-3 K v \delta_{i j}}_{\sigma_{i j}^{L}(\text { Linear })}+\underbrace{2 \mu D_{i j}}_{\sigma_{i j}^{V}(\text { Viscous })} \underbrace{-3 \eta^{v} \dot{v} \delta_{i j}}_{\text {shear }}+\underbrace{2 \eta \dot{D}_{i j}}_{\text {pressure }} \\
\underbrace{+9 K \beta^{v p} v^{2} \delta_{i j}+9 K \beta^{d p} D_{k p} D_{p k} \delta_{i j}}_{\sigma_{i j}^{N L}(\text { Nonlinear })}+\underbrace{4 \mu \beta^{d s} D_{i k} D_{k j}+4 \mu \beta^{c s} v D_{i j}}_{\text {pressure }}
\end{gathered}
$$

The constants $K$ and $\mu$ accompanying nonlinear parameters have been chosen in accordance with (6), as the quadratic power expansion. Four nonlinear parameters of third order (third, in terms of Energy expansion) have been defined and their terms can be interpreted, as follows:

(i) Term with $\beta^{v p}$ : nonlinear volumetric stress generated from the behavior of the volumetric component of the deformation

(ii) Term with $\beta^{d p}$ : nonlinear volumetric stress generated from the behavior of the deviatoric component of the deformation

(iii) Term with $\beta^{d s}$ : nonlinear stress generated from the behavior of the deviatoric component of the deformation

(iv) Term with $\beta^{c s}$ : nonlinear deviatoric stress generated from the interaction between the volumetric and deviatoric components of the deformation

To complete all possible combinations of quadratic terms, there are two additional combinations which have been removed, since the deviatoric trace $D_{k k}$ is always null. (i) A term with $v D_{k k} \delta_{i j}$ : it would be a nonlinear volumetric stress generated from the volumetric part of the deformation

(ii) A term with $D_{k k}^{2} \delta_{i j}$ : with similar interpretation than the previous one

This expression of the stress presents a similar structure to the expression of the stress with Third-Order Elastic Constants in the Landau form $(\mathscr{A}, \mathscr{B}, \mathscr{C})$, shown below in (13), so it will be considered later to obtain the differential equation in a parallel deduction.

Notwithstanding, there is another interesting expression fully separating the nonlinear volumetric and deviatoric components of the stress. The stress term with $\beta^{d s}$ is dependent on the square $D_{i k} D_{k j}$ of the deviatoric component of the deformation $D_{i j}$ and can be split into a volumetric and deviatoric part

$$
\begin{aligned}
D_{i k} D_{k j} & =\frac{1}{3} D_{p k} D_{k p} \delta_{i j}+d_{i j} \\
d_{i j} & =\text { deviatoric }\left[D_{i k} D_{k j}\right]
\end{aligned}
$$

Thus,

$$
\begin{aligned}
& \sigma_{i j}=\underbrace{-3 K v \delta_{i j}}_{\sigma_{i j}^{L}(\text { Linear })}+\underbrace{2 \mu D_{i j}}_{\text {phear }} \underbrace{-3 \eta^{v} v \delta_{i j}}_{\sigma_{i j}^{V}(\text { Viscous })}+\underbrace{2 \eta \dot{D}_{i j}}_{\text {shear }} \\
& \underbrace{\underbrace{+9 K \beta^{v p} v^{2} \delta_{i j}+\left(9 K \beta^{d p}+4 \mu \beta^{d s}\right) D_{k p} D_{p k} \delta_{i j}}_{\text {pressure }}+\underbrace{4 \mu \beta^{d s} d_{i j}+4 \mu \beta^{c s} v D_{i j}}_{\text {shear }}}_{\sigma_{i j}^{N L}(\text { Nonlinear })}
\end{aligned}
$$

2.3. Relationship to Formulations with Landau Constants. As aforementioned, a quite similar expression of the Cauchy stress tensor can be found as a function of the Landau constants and the deformation tensor, for weakly elasticity:

$$
\begin{gathered}
\sigma_{i j}=\underbrace{\lambda \delta_{i j} \varepsilon_{k k}+2 \mu \varepsilon_{i j}}_{\sigma_{i j}^{L}}+\underbrace{2 \eta \dot{\varepsilon}_{i j}-\frac{2}{3} \eta \delta_{i j} \dot{\varepsilon}_{k k}+\eta^{v} \delta_{i j} \dot{\varepsilon}_{k k}}_{\sigma_{i j}^{V}} \\
\underbrace{(\mathscr{A}+4 \mu) \varepsilon_{i k} \varepsilon_{k j}+\mathscr{B} \varepsilon_{k p} \varepsilon_{p k} \delta_{i j}+2\left(\mathscr{B}+K-\frac{5}{3} \mu\right) \varepsilon_{k k} \varepsilon_{i j}+\left(\mathscr{C}-K+\frac{2}{3} \mu\right) \varepsilon_{k k}^{2} \delta_{i j}}_{\sigma_{i j}^{N L}}
\end{gathered}
$$

In order to obtain the relationship between the Landau constants and the new four nonlinear parameters $\beta,(13)$ is separated into volumetric and deviatoric components:

$$
\begin{aligned}
& \varepsilon_{i j}=-v \delta_{i j}+D_{i j} \\
& \varepsilon_{k k}=-v \delta_{k k}+D_{k k}=-3 v \\
& \varepsilon_{i k}=-v \delta_{i k}+D_{i k} \\
& \varepsilon_{k j}=-v \delta_{k j}+D_{k j}
\end{aligned}
$$

$$
\begin{aligned}
\varepsilon_{i k} \varepsilon_{k j} & =v^{2} \delta_{i k} \delta_{k j}-v D_{i k} \delta_{k j}-v D_{k j} \delta_{i k}+D_{i k} D_{k j} \\
\varepsilon_{k p} & =-v \delta_{k p}+D_{k p} \\
\varepsilon_{p k} & =-v \delta_{p k}+D_{p k} \\
\varepsilon_{p k} \varepsilon_{k p} & =v^{2} \delta_{p k} \delta_{k p}-v D_{p k} \delta_{k p}-v D_{k p} \delta_{p k}+D_{p k} D_{k p} \\
\varepsilon_{k k}^{2} & =9 v^{2}
\end{aligned}
$$


The nonlinear terms of (13) result in the following expressions:

$$
\begin{aligned}
& (\mathscr{A}+4 \mu) \varepsilon_{i k} \varepsilon_{k j} \\
& =(\mathscr{A}+4 \mu)\left(v^{2} \delta_{i k} \delta_{k j}-v D_{i k} \delta_{k j}-v \delta_{i k} D_{k j}+D_{i k} D_{k j}\right) \\
& \mathscr{B} \varepsilon_{k p} \varepsilon_{p k} \delta_{i j}=\mathscr{B}\left(3 v^{2}+D_{k p} D_{p k}\right) \delta_{i j} \\
& 2\left(\mathscr{B}+K-\frac{5}{3} \mu\right) \varepsilon_{k k} \varepsilon_{i j} \\
& =2\left(\mathscr{B}+K-\frac{5}{3} \mu\right)\left(3 v^{2} \delta_{i j}-3 v D_{i j}\right) \\
& \left(C-K+\frac{2}{3}\right) \varepsilon_{k k}^{2} \delta_{i j}=\left(C-K+\frac{2}{3} \mu\right) 9 v^{2} \delta_{i j}
\end{aligned}
$$

The above analysis is also valid by combining the nonlinear part of the stress with $v$ and $D_{i j}$ in the constitutive equation,

$$
\begin{aligned}
\sigma_{i j}^{N L}= & (\mathscr{A}+9 \mathscr{B}+9 \mathscr{C}-3 K) v^{2} \delta_{i j}+\mathscr{B} D_{k p} D_{p k} \delta_{i j} \\
& +(\mathscr{A}+4 \mu) D_{i k} D_{k j} \\
& +(-2 \mathscr{A}+6 \mathscr{B}-6 K+2 \mu) v D_{i j}
\end{aligned}
$$

Comparing this expression with (9),

$$
\begin{aligned}
\beta^{v p} & =\frac{\mathscr{A}+9 \mathscr{B}+9 \mathscr{C}-3 K}{9 K} \\
\beta^{d p} & =\frac{\mathscr{B}}{9 K} \\
\beta^{d s} & =\frac{\mathscr{A}+4 \mu}{4 \mu} \\
\beta^{c s} & =\frac{-2 \mathscr{A}-6 \mathscr{B}-6 K+2 \mu}{4 \mu} \\
\mathscr{A} & =4 \mu\left(\beta^{d s}-1\right) \\
\mathscr{B} & =9 K \beta^{d p} \\
\mathscr{C} & =K \beta^{v p}-\frac{4}{9} \mu \beta^{d s}-9 K \beta^{d p}+\frac{1}{3} K
\end{aligned}
$$

For the viscous components, it is shown that the establish definition in (6) matches Landau's one:

$$
\begin{aligned}
2 \eta \dot{\varepsilon}_{i j} & =2 \eta\left(-\dot{v} \delta_{i j}+\dot{D}_{i j}\right) \\
-\frac{2}{3} \eta \dot{\varepsilon}_{k k} \delta_{i j} & =2 \eta \dot{v} \delta_{i j} \\
\eta^{v} \dot{\varepsilon}_{k k} \delta_{i j} & =-3 \eta^{v} \dot{v} \delta_{i j} \\
\sigma_{i j}^{V} & =-3 \eta^{v} \dot{v} \delta_{i j}+2 \eta \dot{D}_{i j}
\end{aligned}
$$

2.4. Differential Wave Equation. Adopting the acoustic nonlinear constitutive equation presented in (9), in terms of deviatoric a volumetric parts of the deformation, it is possible to obtain the three dimensional nonlinear equation of motion in terms of the new four parameters $\beta$. Previously, the kinematic relations must be introduced in the constitutive equation.

$$
\varepsilon_{i j}=\frac{1}{2}\left(\frac{\partial u_{i}}{\partial x_{j}}+\frac{\partial u_{j}}{\partial x_{i}}+\frac{\partial u_{k}}{\partial u_{i}} \frac{\partial u_{k}}{\partial u_{j}}\right)
$$

The wave equation is,

$$
\begin{aligned}
& \rho \frac{\partial^{2} u_{i}}{\partial t^{2}}=K\left(\frac{\partial^{2} u_{k}}{\partial x_{k} \partial x_{i}}+\frac{\partial u_{l}}{\partial x_{k}} \frac{\partial^{2} u_{l}}{\partial x_{k} \partial x_{i}}\right) \\
& +\mu\left(\frac{\partial^{2} u_{i}}{\partial x_{j}^{2}}+\frac{\partial^{2} u_{j}}{\partial x_{i} \partial x_{j}}+\frac{\partial^{2} u_{k}}{\partial x_{j}^{2}} \frac{\partial u_{k}}{\partial x_{i}}+\frac{\partial^{2} u_{k}}{\partial x_{i} \partial x_{j}} \frac{\partial u_{k}}{\partial x_{j}}\right. \\
& \left.-\frac{2}{3}\left(\frac{\partial^{2} u_{k}}{\partial x_{k} \partial x_{i}}+\frac{\partial u_{l}}{\partial x_{k}} \frac{\partial^{2} u_{l}}{\partial x_{k} \partial x_{i}}\right)\right) \\
& +2 K \beta^{v p}\left(\frac{\partial^{2} u_{k}}{\partial x_{k} \partial x_{i}} \frac{\partial u_{l}}{\partial x_{l}}\right) \\
& +9 K \beta^{d p}\left(\frac { 1 } { 2 } \left(\frac{\partial^{2} u_{l}}{\partial x_{k} \partial x_{i}} \frac{\partial u_{l}}{\partial x_{k}}+\frac{\partial^{2} u_{k}}{\partial x_{l} \partial x_{i}} \frac{\partial u_{k}}{\partial x_{l}}\right.\right. \\
& \left.\left.+\frac{\partial^{2} u_{l}}{\partial x_{k} \partial x_{i}} \frac{\partial u_{k}}{\partial x_{l}}+\frac{\partial^{2} u_{k}}{\partial x_{l} \partial x_{i}} \frac{\partial u_{l}}{\partial x_{k}}\right)-\frac{2}{3} \frac{\partial^{2} u_{k}}{\partial x_{k} \partial x_{i}} \frac{\partial u_{l}}{\partial x_{l}}\right) \\
& +4 \mu \beta^{d s}\left(\frac { 1 } { 4 } \left(\frac{\partial^{2} u_{i}}{\partial x_{k} \partial x_{j}} \frac{\partial u_{k}}{\partial x_{j}}+\frac{\partial^{2} u_{k}}{\partial x_{i} \partial x_{j}} \frac{\partial u_{k}}{\partial x_{j}}\right.\right. \\
& \left.\left.+\frac{\partial^{2} u_{i}}{\partial x_{k} \partial x_{j}} \frac{\partial u_{j}}{\partial x_{k}}+\frac{\partial^{2} u_{k}}{\partial x_{i} \partial x_{j}} \frac{\partial u_{j}}{\partial x_{k}}\right)\right) \\
& +4 \mu \beta^{d s}\left(\frac { 1 } { 4 } \left(\frac{\partial^{2} u_{k}}{\partial x_{j} \partial x_{j}} \frac{\partial u_{i}}{\partial x_{k}}+\frac{\partial^{2} u_{j}}{\partial x_{k} \partial x_{j}} \frac{\partial u_{i}}{\partial x_{k}}\right.\right. \\
& \left.\left.+\frac{\partial^{2} u_{k}}{\partial x_{j} \partial x_{j}} \frac{\partial u_{k}}{\partial x_{i}}+\frac{\partial^{2} u_{j}}{\partial x_{k} \partial x_{j}} \frac{\partial u_{k}}{\partial x_{i}}\right)\right) \\
& +4 \mu \beta^{d s}\left(-\frac{1}{3}\left(\frac{\partial^{2} u_{k}}{\partial x_{k} \partial x_{j}} \frac{\partial u_{i}}{\partial x_{j}}+\frac{\partial^{2} u_{k}}{\partial x_{k} \partial x_{j}} \frac{\partial u_{j}}{\partial x_{i}}\right.\right. \\
& \left.\left.+\frac{\partial^{2} u_{i}}{\partial x_{j}^{2}} \frac{\partial u_{k}}{\partial x_{k}}+\frac{\partial^{2} u_{j}}{\partial x_{i} \partial x_{j}} \frac{\partial u_{k}}{\partial x_{k}}\right)+\frac{2}{9} \frac{\partial^{2} u_{k}}{\partial x_{k} \partial x_{i}} \frac{\partial u_{l}}{\partial x_{l}}\right) \\
& +4 \mu \beta^{d s}\left(-\frac{1}{6}\left(\frac{\partial^{2} u_{l}}{\partial x_{k} \partial x_{i}} \frac{\partial u_{l}}{\partial x_{k}}+\frac{\partial^{2} u_{k}}{\partial x_{l} \partial x_{i}} \frac{\partial u_{k}}{\partial x_{l}}\right.\right.
\end{aligned}
$$




$$
\begin{aligned}
& \left.\left.+\frac{\partial^{2} u_{l}}{\partial x_{k} \partial x_{i}} \frac{\partial u_{k}}{\partial x_{l}}+\frac{\partial^{2} u_{k}}{\partial x_{l} \partial x_{i}} \frac{\partial u_{l}}{\partial x_{k}}\right)+\frac{2}{9} \frac{\partial^{2} u_{k}}{\partial x_{k} \partial x_{i}} \frac{\partial u_{l}}{\partial x_{l}}\right) \\
& +4 \mu \beta^{c s}\left(-\frac{1}{6}\left(\frac{\partial^{2} u_{k}}{\partial x_{k} \partial x_{j}} \frac{\partial u_{i}}{\partial x_{j}}+\frac{\partial^{2} u_{k}}{\partial x_{k} \partial x_{j}} \frac{\partial u_{j}}{\partial x_{i}}\right.\right. \\
& \left.\left.+\frac{\partial^{2} u_{i}}{\partial x_{j}^{2}} \frac{\partial u_{k}}{\partial x_{k}}+\frac{\partial^{2} u_{j}}{\partial x_{i} \partial x_{j}} \frac{\partial u_{k}}{\partial x_{k}}\right)+\frac{2}{9} \frac{\partial^{2} u_{k}}{\partial x_{k} \partial x_{i}} \frac{\partial u_{l}}{\partial x_{l}}\right)
\end{aligned}
$$

where, as it was defined, $K$ is the Bulk modulus, $\mu$ is the shear modulus, $\rho$ is the density, and $\beta^{v p}, \beta^{d p}, \beta^{d s}$, and $\beta^{c s}$ are the four nonlinear parameters of third order already explained in the constitutive expression.

\section{Discussion}

Considering the new nonlinear constitutive equation (12), it can be applied to the case of a pure plane horizontal shear wave. Disregarding the viscous term, the deformation tensor $\varepsilon_{i j}$ will have all components null but $\varepsilon_{12}$, yielding $D_{i j}=\varepsilon_{i j}$, $v=-(1 / 3) \varepsilon_{k k}=0$, and $D_{k p} D_{p k}=2 \varepsilon_{12}^{2}$,

$$
\begin{gathered}
\sigma_{i j}=\underbrace{\left[\begin{array}{ccc}
0 & 2 \mu \varepsilon_{12} & 0 \\
2 \mu \varepsilon_{12} & 0 & 0 \\
0 & 0 & 0
\end{array}\right]}_{\text {linear }} \\
+\underbrace{\left[\begin{array}{ccc}
\left(9 K \beta^{d p} 2+4 \mu \beta^{c s}\right) \varepsilon_{12}^{2} & 0 & 0 \\
0 & \left(9 K \beta^{d p} 2+4 \mu \beta^{c s}\right) \varepsilon_{12}^{2} & 0 \\
0 & 9 K \beta^{d p} 2 \varepsilon_{12}^{2}
\end{array}\right]}_{\text {nonlinear }}
\end{gathered}
$$

In this case, the linear part of the stress continues with the same shear structure while the nonlinear part is a diagonal matrix meaning that there exists a volumetric component in it that would contribute to posterior volumetric deformations. Only two constants survive reducing the mathematical complexity for shear waves.

If it is a compressional wave in the $x_{1}$ direction, with $\varepsilon_{i j}=$ $0 \forall i j \neq 11$,

$$
\begin{aligned}
& \sigma_{i j}=\underbrace{\left[\begin{array}{ccc}
\frac{2}{3} \varepsilon_{11} & 0 & 0 \\
0 & -\frac{1}{3} \delta_{11}+2 \mu & 0 \\
0 & 0 & -\frac{1}{3} \varepsilon_{11}
\end{array}\right]}_{\text {linear }} \\
& +\underbrace{\left(K \beta^{v p}+6 K \beta^{d p}+4 \mu \beta^{c s}\right) \varepsilon_{11}^{2} \delta_{i j}+4 \mu\left(\beta^{d s}-\beta^{c s}\right)\left[\begin{array}{ccc}
\frac{2}{9} \varepsilon_{11}^{2} & 0 & 0 \\
0 & -\frac{1}{9} \varepsilon_{11}^{2} & 0 \\
0 & 0 & -\frac{1}{9} \varepsilon_{11}^{2}
\end{array}\right]}_{\text {novlinear }},
\end{aligned}
$$

where now the four nonlinear coefficients must be considered, indicating the existence of all kind of cross and parallel effects, although pure volumetric plus deviatoric nonlinear stress components are the result, allowing the separation of both nonlinear components.

Landau constants only account for the constitutive nonlinear behavior of the material, expressed in all the nonlinear terms of (13) that include $\mathscr{A} \mathscr{B}$ and $\mathscr{C}$. As mentioned above, there are several additional nonlinear terms in this expression without these constants, but with $K$ and $\mu$. They represent the geometrical nonlinearity originated in the geometrical transformation from the Piola tensor $P_{i j}$ as a function of the strain energy $U$ to get the Cauchy stress tensor.

$$
P_{i j}=\frac{\partial U}{\partial F_{i j}}=F_{i j} \frac{\partial U}{\partial \varepsilon_{i j}}
$$

$$
\begin{aligned}
\sigma_{i j} & =\frac{1}{\operatorname{det}(F)} P_{i j} F_{j i} \\
\sigma_{i j} & =\frac{1}{\operatorname{det}(F)} F_{i j} \frac{\partial U}{\partial \varepsilon_{i j}} F_{j i}
\end{aligned}
$$

It must be noted that the four new $\beta$ constants would include constitutive and geometrical effects, with the exception of $\beta^{d p}$ that is only of constitutive nature (see (29) and (28), (30), and (31)).

Considering the relations among the four new $\beta$ parameters and Landau constants in (32), (33), (34), (28), (29), (30), and (31), some conclusions can be extracted.

(i) As the Landau constant $\mathscr{B}$ is only dependent on $\beta^{d p}$ (see (33)), $\mathscr{B}$ is a measure of the intensity on the cross effect from the deviatoric deformation into a nonlinear volumetric stress. 
(ii) As the Landau constant $\mathscr{A}$ is only dependent on $\beta^{d s}$ (see (32)), $\mathscr{A}$ is a measure of the intensity on the nonlinear effect from the deviatoric deformation into the stress, which can be split into both volumetric and deviatoric parts (see (12)).

(iii) As the Landau constant $\mathscr{C}$ is only dependent on $\beta^{v p}$ (see (34)), $\mathscr{C}$ is the only one that measures the intensity on the nonlinear effect from the volumetric deformation into the volumetric component of the stress.

(iv) As expected, $\beta^{c s}$ is linearly dependent on $\beta^{d s}$ and $\beta^{d p}$ :

$$
\beta^{c s}=-2 \beta^{d s}-\frac{27 K}{2 \mu} \beta^{d p}-\frac{1}{2}-\frac{3 K}{2 \mu}
$$

\section{Conclusions}

A new approach is proposed in the field of nonlinear acoustics introducing the volumetric and deviatoric separation in deformation and stress. The relevance of this expression is directly linked to the possibility of separating longitudinal and shear waves and selecting the responses of interest. Additional interpretations on the Landau constants are deducted in terms of the cross and parallel effects between volumetric and deviatoric components.

\section{Data Availability}

No data were used to support this study.

\section{Conflicts of Interest}

The authors declare that there are no conflicts of interest regarding the publication of this article.

\section{Acknowledgments}

This research was supported by the Ministry of Education [Grant nos. DPI2014-51870-R, DPI2017-85359-R, and UNGR15-CE-3664], Ministry of Health [DTS15/00093 and PI16/00339], Junta de Andalucía [PIN-0030-2017 and PI0107-2017 projects], and University of Granada [PP2017PIP2019].

\section{References}

[1] M. F. Hamilton and D. T. Blackstock, Nonlinear Acoustics, vol. 237, Academic Press, San Diego, Calif, USA, 1998.

[2] D. D. Muir, One-Sided Ultrasonic Determination of Third Order Elastic Constants Using Angle-Beam Acoustoelasticity Measurements [Dissertation thesis], Georgia Institute of Technology, 2009.

[3] C. Pantea, C. F. Osterhoudt, and D. N. Sinha, "Determination of acoustical nonlinear parameter $\beta$ of water using the finite amplitude method," Ultrasonics, vol. 53, no. 5, pp. 1012-1019, 2013.

[4] C. Trarieux, S. Callé, H. Moreschi, G. Renaud, and M. Defontaine, "Modeling nonlinear viscoelasticity in dynamic acoustoelasticity," Applied Physics Letters, vol. 105, no. 26, Article ID 264103, 2014.

[5] J. Melchor, L. Peralta, G. Rus, N. Saffari, and J. Soto, "Singletransmitter setup on nonlinear mixing to measure acoustic nonlinearity of first order," in Proceedings of the 6th European Symposium on Ultrasonic Characterization of Bone, (ESUCB '15), pp. 1-4, IEEE, 2015.

[6] R. Munoz, G. Rus, N. Bochud et al., "Nonlinear ultrasonics for early damage detection," Emerging Design Solutions in Structural Health Monitoring Systems, p. 171, 2015.

[7] N. Antonin, M. Bentahar, V. Tournat et al., "Nonlinear acoustic characterization of micro-damaged materials through higher harmonic resonance analysis," NDT \& E International, vol. 45, no. 1, pp. 1-8, 2012.

[8] Y. Jiang, G.-Y. Li, L.-X. Qian et al., "Characterization of the nonlinear elastic properties of soft tissues using the supersonic shear imaging (SSI) technique: Inverse method, ex vivo and in vivo experiments," Medical Image Analysis, vol. 20, no. 1, pp. 97 111, 2015.

[9] M. McGovern and H. Reis, "Linear and nonlinear ultrasonic characterization of in limestone," in Proceedings of the SPIE Smart Structures and Materials \& Nondestructive Evaluation and Health Monitoring, p. 94381A, San Diego, Calif, USA.

[10] R. Munoz, J. C. Ruano, G. Rus et al., "Nonlinear ultrasonics for early damage detection," Emerging Design Solutions in Structural Health Monitoring Systems, pp. 171-206, 2015.

[11] K. E. A. Van Den Abeele, W. Desadeleer, G. De Schutter, and M. Wevers, "Active and passive monitoring of the early hydration process in concrete using linear and nonlinear acoustics," Cement and Concrete Research, vol. 39, no. 5, pp. 426-432, 2009.

[12] J. Kim and J. Lee, "A micromechanical model for nonlinear acoustic properties of interfaces between solids," Journal of Applied Physics, vol. 101, no. 4, Article ID 043501, 2007.

[13] S. S. Kessler, S. M. Spearing, and C. Soutis, "Damage detection in composite materials using Lamb wave methods," Smart Materials \& Structures, vol. 11, no. 2, pp. 269-278, 2002.

[14] L. D. Landau and E. M. Lifshitz, “Theory of elasticity," in Course of Theoretical Physics, vol. 7, 1986.

[15] F. D. Murnaghan, Finite Deformation of an Elastic Solid, Chapman \& Hall, London, UK, 1951.

[16] M. A. Breazeale and D. O. Thompson, "Finite-amplitude ultrasonic waves in aluminum," Applied Physics Letters, vol. 3, no. 5, pp. 77-78, 1963.

[17] J. Melchor, Mechanics of Nonlinear Ultrasound in Tissue, University of Granada, 2016.

[18] L. K. Zarembo and V. A. Krasil'Nikov, "Nonlinear phenomena in the propagation of elastic waves in solids," Soviet Physics-Uspekhi, vol. 13, no. 6, pp. 778-797, 1971.

[19] R. A. Guyer, J. Ten Cate, and P. Johnson, "Hysteresis and the dynamic elasticity of consolidated granular materials," Physical Review Letters, vol. 82, no. 16, pp. 3280-3283, 1999.

[20] K. E. A. Van Den Abeele, P. A. Johnson, R. A. Guyer, and K. R. McCall, "On the quasi-analytic treatment of hysteretic nonlinear response in elastic wave propagation," The Journal of the Acoustical Society of America, vol. 101, no. 4, pp. 1885-1898, 1997.

[21] J. Rivière, P. Shokouhi, R. A. Guyer, and P. A. Johnson, "A set of measures for the systematic classification of the nonlinear elastic behavior of disparate rocks," Journal of Geophysical Research: Solid Earth, vol. 120, no. 3, pp. 1587-1604, 2015. 
[22] K. E. A. Van Den Abeele, P. A. Johnson, and A. Sutin, "Nonlinear elastic wave spectroscopy (NEWS) techniques to discern material damage, Part I: Nonlinear wave modulation spectroscopy (NWMS)," Research in Nondestructive Evaluation, vol. 12, no. 1, pp. 17-30, 2000.

[23] K. E. A. Van Den Abeele, J. Carmeliet, J. A. Ten Cate, and P. A. Johnson, "Nonlinear elastic wave spectroscopy (NEWS) techniques to discern material damage, Part II: Single-mode nonlinear resonance acoustic spectroscopy," Research in Nondestructive Evaluation, vol. 12, no. 1, pp. 31-42, 2000.

[24] L. D. Landau and E. M. Lifshitz, Theory of Elasticity, Pergamon Press, Oxford, UK, 1986.

[25] G. Rus, "Nature of acoustic nonlinear radiation stress," Applied Physics Letters, vol. 105, no. 12, Article ID 121904, 2014. 


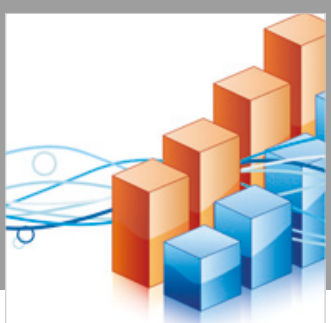

Advances in

Operations Research

\section{-n-m}
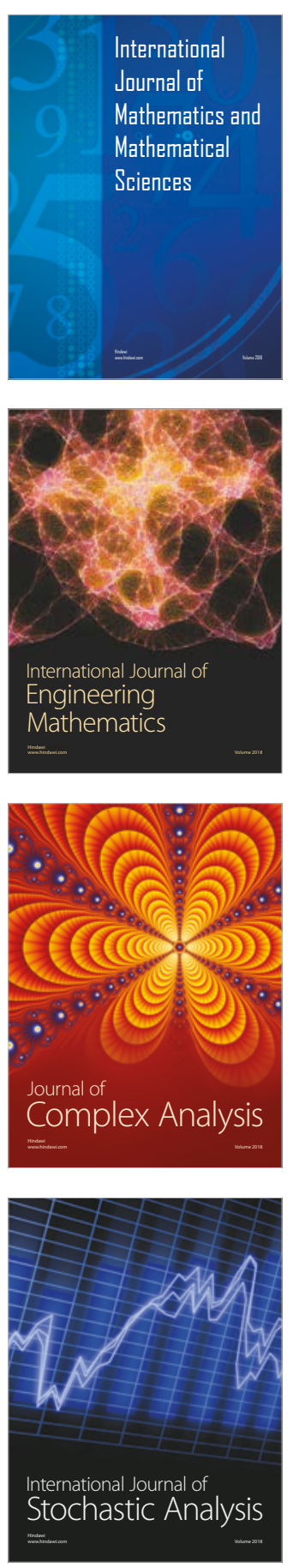
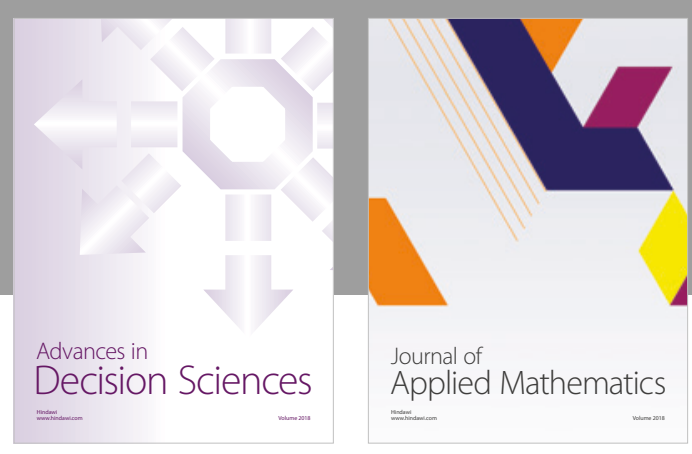

Journal of

Applied Mathematics
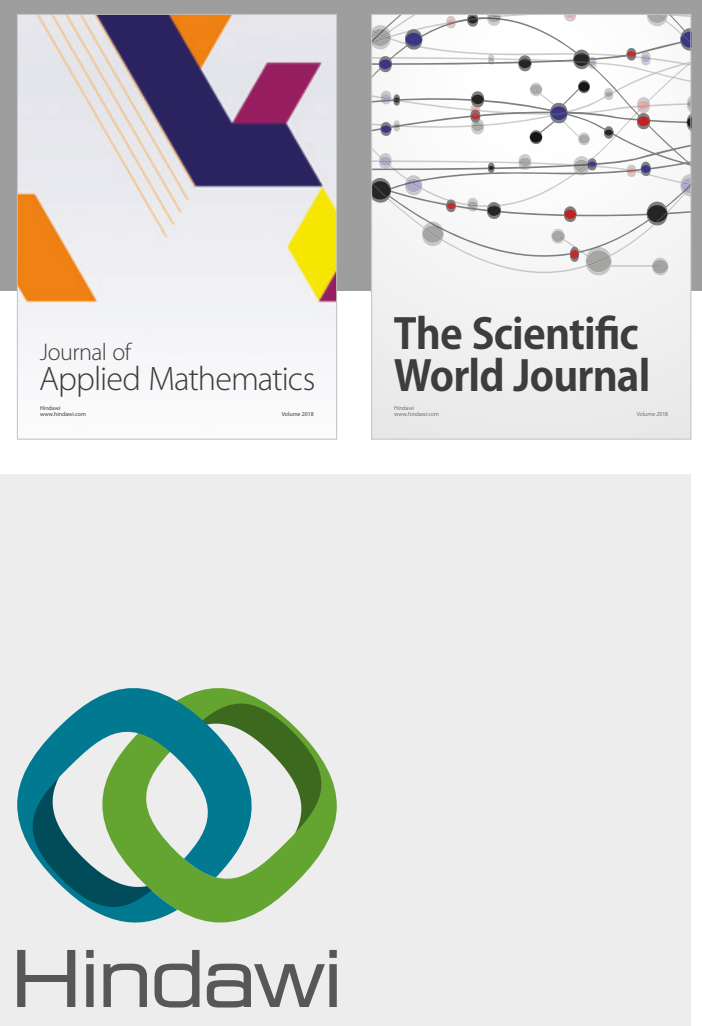

Submit your manuscripts at

www.hindawi.com

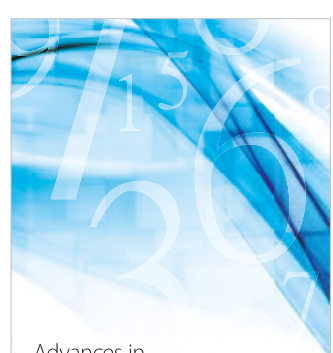

Advances in
Numerical Analysis
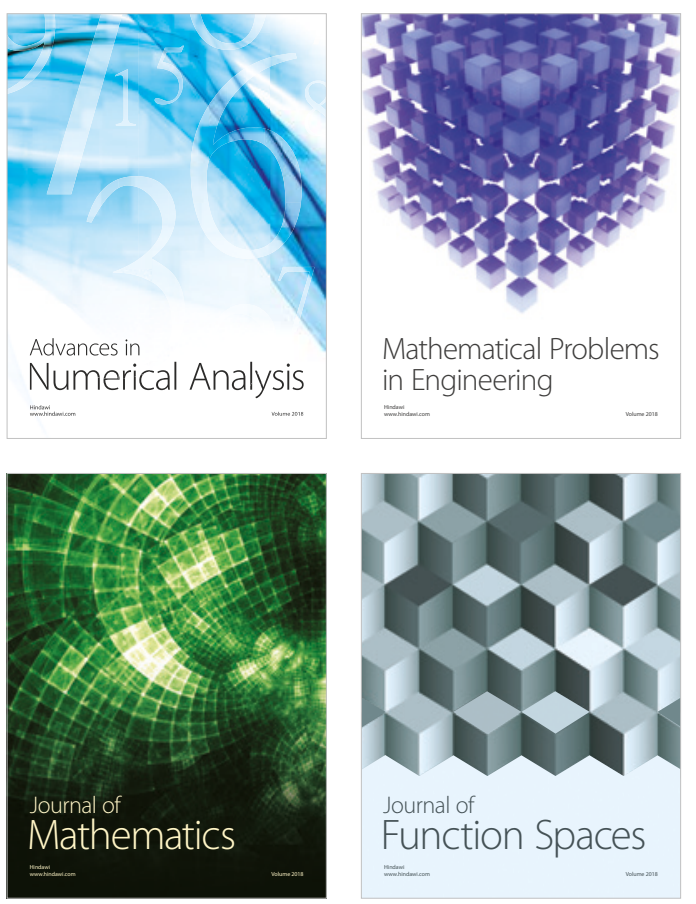

Mathematical Problems in Engineering

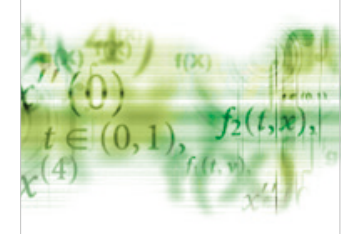

International Journal of

Differential Equations

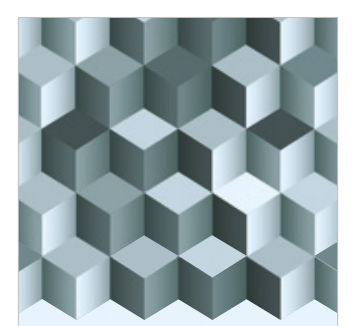

Journal of

Function Spaces

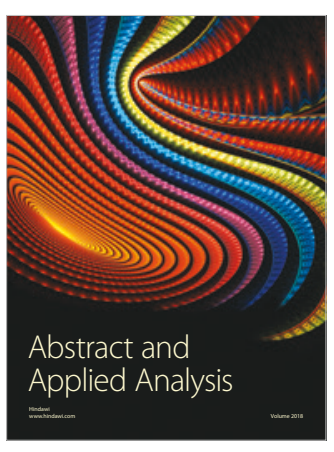

The Scientific

World Journal

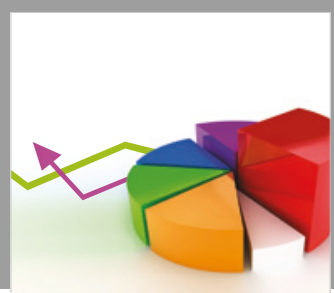

Journal of

Probability and Statistics
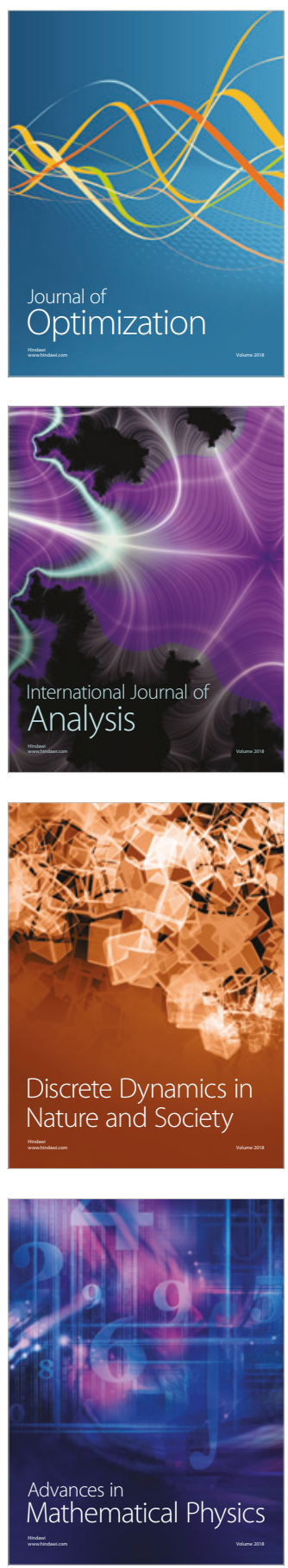